\title{
Urine biomarkers for monitoring acute kidney injury in premature infants
}

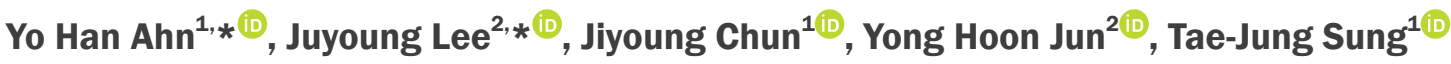 \\ 'Department of Pediatrics, Hallym University Kangnam Sacred Heart Hospital, Seoul, Republic of Korea \\ ${ }^{2}$ Department of Pediatrics, Inha University Hospital, Incheon, Republic of Korea
}

\begin{abstract}
Background: Premature infants are at high risk for acute kidney injury (AKI). Serum creatinine (Cr) has limitations for evaluating kidney function in premature infants. We evaluated whether urine biomarkers could be used to monitor AKI in premature infants.

Methods: A prospective cohort study was conducted among infants born at $<37$ weeks. Urine biomarkers and serum $\mathrm{Cr}$ were measured on postnatal days 1, 3, 5, 7, 10, and 14. Infants were divided into 3 groups according to gestational age $(\mathrm{GA}) ;<28,28$ to $<32$ and 32 to $<37$ weeks.

Results: AKI occurred in 17 of $83(20.5 \%)$ recruited infants at a median age of 7 (interquartile range 5-10) days. While the most common cause of AKI was hemodynamically significant patent ductus arteriosus (53.8\%) in infants of GA $<28$ weeks, necrotizing enterocolitis was the leading cause (50.0\%) in infants of GA 28 to $<32$ weeks. Urinary levels of neutrophil-gelatinase-associated lipocalin/Cr were higher and epidermal growth factor/Cr were lower in AKI group before the onset of AKI in infants of GA $<28$ weeks. In infants of GA 28 to $<32$ weeks, urinary interleukin- $8 / C r$ levels were higher in AKI group at approximately the time of AKI onset.

Conclusion: Several urine biomarkers were significantly different between AKI and no AKI groups, and some had changed before the onset of AKI. These groups were distinct according to causative factors of AKI and GA. Urine biomarkers could be useful for monitoring the development of AKI in premature infants.
\end{abstract}

Keywords: Acute kidney injury, Biomarker, Gestational age, Premature infants, Urine

Received March 20, 2020; Revised June 13, 2020;

Accepted June 14, 2020

Editor: Hee Gyung Kang, Seoul National University, Seoul, Republic of Korea

Correspondence: Tae-Jung Sung

Department of Pediatrics, Hallym University Kangnam Sacred Heart Hospital, 1 Singil-ro, Yeongdeungpo-gu, Seoul 07441, Republic of Korea. E-mail: neosung@hallym.or.kr

*Yo Han Ahn and Juyoung Lee contributed equally to this study. Yo Han Ahn's current affiliation: Department of Pediatrics, Seoul National University Children's Hospital, Seoul, Republic of Korea

Copyright @ 2020 by The Korean Society of Nephrology

(a) This is an open-access article distributed under the terms of the Creative Commons Attribution Non-Commercial License (http://creativecommons. org/licenses/by-nc-nd/4.0/), which permits unrestricted non-commercial use, distribution, and reproduction in any medium, provided the original work is properly cited.

\section{Introduction}

The survival of premature infants has improved substantially over recent decades. Increased survival of preterm newborns has raised concern regarding increased morbidities. Postnatal complications for premature infants include abnormal kidney function and, in severe cases, kidney failure [1]. Acute kidney injury (AKI) is defined as a sudden decline in kidney function that results in imbalances in fluid levels, electrolytes, and waste products. Recent studies have shown that AKI occurs in $12 \%$ to $40 \%$ of premature infants and is associated with poor outcomes [1-3].

Obviously, premature infants are vulnerable to kidney injury because of their immature kidney structure and function. Additionally, aspects of the extrauterine envi- 
ronment, which are experienced indirectly during fetal development, are hostile and detrimental to optimal nephrogenesis, resulting in incomplete glomeruli and tubules. The negative effects include hypoxic damage, sepsis, the presence of nephrotoxic agents, necrotizing enterocolitis (NEC), and patent ductus arteriosus (PDA) $[1,2,4-6]$.

Premature infants have decreased nephron endowment, which results in low potency to reverse abnormal kidney function [7]. It has been suggested that low birth weight and preterm birth are correlated with microalbuminuria, reduced glomerular filtration rate, hypertension, and focal segmental glomerulosclerosis later in life [8-10]. Therefore, early detection of AKI is important to prevent the progression of abnormal kidney function in these vulnerable subjects. Currently, the diagnosis of AKI is based on an increase in serum creatinine ( $\mathrm{Cr}$ ) and/or decrease in urine output. However, serum $\mathrm{Cr}$ level has limitations for evaluating kidney function in premature infants because neonatal serum $\mathrm{Cr}$ reflects maternal levels during the early postnatal period, and these levels decline over days to weeks rather than maintaining a steady state [11]. On the other hand, preterm newborns often have non-oliguric AKI, making oliguria an insensitive marker for the early detection of kidney injury. The purpose of this study was to identify useful urine biomarkers to predict AKI in premature infants.

\section{Methods}

\section{Study participants}

A prospective cohort study was conducted in a sample of premature infants born at less than gestational age (GA) 37 weeks and admitted to the neonatal intensive care units of two tertiary hospitals during the period from March 2016 to February 2017. This study is registered at ClinicalTrials.gov (NCT02813109). Exclusion criteria included the following: congenital heart anomalies except for PDA, congenital anomalies of the kidney, and transfer from another hospital. Neonates with congenital heart or kidney anomalies are at high risk for AKI because of their physiological vulnerabilities, so that they do not follow the natural course of premature infants. For analyses, infants were divided to 3 groups according to GA following the World Health Organization classification: $<28$ weeks (extremely preterm), 28 to $<32$ weeks (very preterm), and 32 to $<37$ weeks (moderate to late preterm). This study was approved by the Institutional Review Boards of Kangnam Sacred Heart Hospital (IRB No. 2016-05-54) and Inha University Hospital (IRB No. 2016-04-002), and we obtained parental informed consent.

\section{Laboratory evaluations and clinical information}

Serum $\mathrm{Cr}$ and electrolytes were measured on postnatal days $1,3,5,7,10$, and 14; isotope dilution mass spectrometry was used to measure serum concentrations of $\mathrm{Cr}$ and indirect ion selective electrodes were used for assessing serum electrolytes at both hospitals. If venous blood sampling was not required, these values were checked using the iSTAT ${ }^{\circledR}$ CHEM8+ (Abbott, Abbott Park, IL, USA) with capillary blood samples. The lowest serum $\mathrm{Cr}$ values after the 2 nd day of life were defined as the baseline levels. AKI was classified using the neonatal modified Kidney Disease Improving Global Outcomes (KDIGO) definition [6]. We collected demographic, clinical, laboratory, and outcome data for infants and mothers before birth and during hospitalization. Neonatal severity of illness on neonatal intensive care unit admission was assessed using Apgar scores and clinical risk index for babies (CRIB) II scores [12]. Pathologic features of chorioamnionitis and placental infarction were collected if placental biopsy was performed.

\section{Urine biomarker assays}

To measure the concentrations of biomarkers, $1-3 \mathrm{~mL}$ of urine was collected at 1, 3, 5, 7, 10, and 14 days of life. Urine specimens were centrifuged, aliquoted, and stored at $-70^{\circ} \mathrm{C}$ until assays were performed. A multiplex Luminex assay ${ }^{\circledR}$ (R\&D Systems, Inc., Minneapolis, MN, USA) was used to measure urine biomarkers, which included neutrophil-gelatinase-associated lipocalin (NGAL), interleukin-8 (IL-8), cystatin C, epidermal growth factor (EGF), liver fatty acid binding protein (L-FABP), osteopontin (OPN), and kidney injury molecule-1 (KIM-1). Simultaneously, urine Cr levels were measured using the Cell Biolab Urinary Creatinine Assay Kit (Cell Biolabs, Inc., San Diego, CA, USA). All values were adjusted with urine Cr. 


\section{Statistical analysis}

Statistical analyses were performed using IBM SPSS version 23.0 (IBM Corp., Armonk, NY, USA). Analyses of clinical data were performed on an intention-to-treat basis, and biochemical data for urine from subjects who completed the protocol were analyzed. We analyzed the results using non-parametric tests due to small sample size. Categorical variables were analyzed using Fisher's exact test, and continuous variables were compared using the Mann-Whitney $U$ test. Multivariate logistic regression analysis was performed with adjustments for factors that had $P<0.20$ in the univariate analysis. Differences in biomarker levels according to the GA were analyzed using the Kruskal-Wallis test. A $P$ value $<0.05$ was considered significant.

\section{Results}

A total of 105 preterm infants were screened for the study, and 22 were excluded due to refusal to participate $(\mathrm{n}=16)$ or skin problems that made it impossible to collect urine samples $(n=6)$. Eighty-three preterm infants were enrolled. Their median GA and birth weight were 30.7 (interquartile range [IQR], 28.5-33.3) weeks and 1,420 (IQR 1,105-1,860) g, respectively. Urine biomarkers were evaluated in 81 infants, excluding 2 patients for whom we failed to collect urine samples because of skin injuries caused by the attachment of a urine bag (Fig. 1).

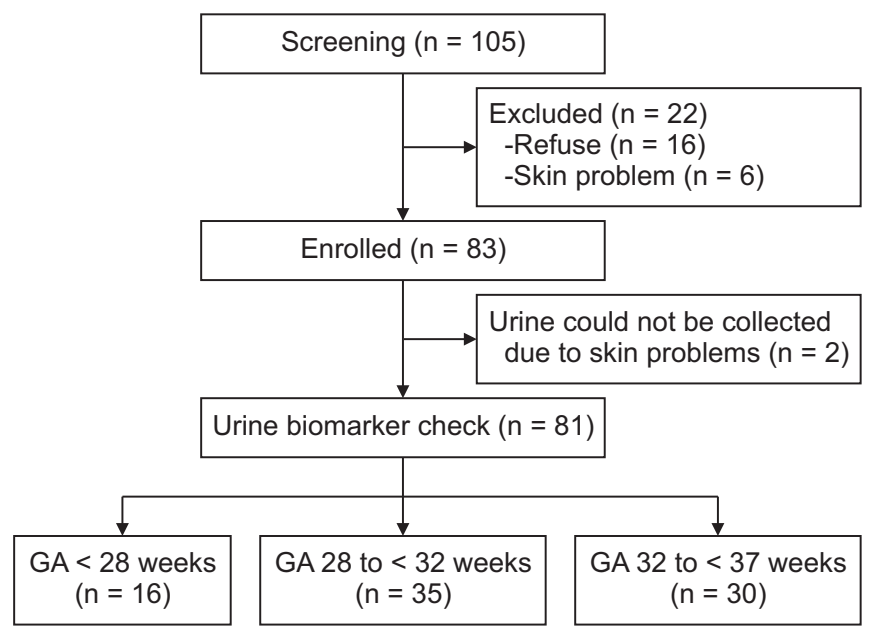

Figure 1. Study participation.

GA, gestational age.
AKI occurrence and clinical course

AKI occurred in 17 (20.5\%) infants at a median age of 7 (IQR 5-10) days. AKI incidence varied inversely with GA: $72.2 \%(\mathrm{n}=13)$ for $\mathrm{GA}<28$ weeks, $11.4 \%(\mathrm{n}=4)$ for GA 28 to $<32$ weeks, and $0 \%$ for GA 32 to $<37$ weeks. Among infants born before GA 28 weeks, infants with AKI had lower incidence of maternal diabetes, lower GA and higher CRIB II scores than those without AKI in the univariate analysis. For infants of GA 28 to $<32$ weeks, only birth weight was associated with AKI in the univariate analysis. However, there were no significant differences for any of the above factors in the multivariate regression analysis (Table 1).

According to the neonatal modified KDIGO definition, $10(12.0 \%)$ infants were classified as stage $1,5(6.0 \%)$ as stage 2 , and $2(2.4 \%)$ as stage 3 (Table 2). No patients required kidney replacement therapy during the study period. While the most common cause of AKI was hemodynamically significant PDA (53.8\%) in infants of GA < 28 weeks, NEC was the leading cause $(50.0 \%)$ in infants of GA 28 to $<32$ weeks $(P=0.053)$. The length of hospital stay was not different between the two groups among infants of GA < 28 weeks (no AKI vs. AKI group, median 91 [IQR, 79-99] vs. 96 [85-105] days, $P=0.775)$. However, length of stay was significantly longer in the AKI group than in no AKI group among infants of GA 28 to $<32$ weeks (no AKI vs. AKI group, median 47 [IQR, 42-57] vs. 82 [70-107] days, $P=0.001$ ) (Table 1). Infants with AKI were discharged at a median 39.7 (IQR, 38.3-41.3) weeks of postmenstrual age with estimated glomerular filtration rate of median 97.1 (IQR, 60.5-109.0) $\mathrm{mL} / \mathrm{min} / 1.73 \mathrm{~m}^{2}$. While none of them had reduced GFR or abnormal findings on ultrasonography, $30.7 \%$ had proteinuria defined as urine protein/Cr ratio greater than $0.5 \mathrm{mg} / \mathrm{mg}$ or $2+$ or higher on a urine dipstick at discharge (Table 2). Patients with proteinuria could be categorized as having chronic kidney disease following AKI [13].

\section{Urine biomarkers}

In infants of GA $<28$ weeks, serum Cr levels of AKI groups were significantly higher from day 5 to day 14 compared to those in the no AKI groups (Fig. 2). The levels of urine NGAL/Cr were significantly higher in the AKI group than in the no AKI group from day 3 to day 7 


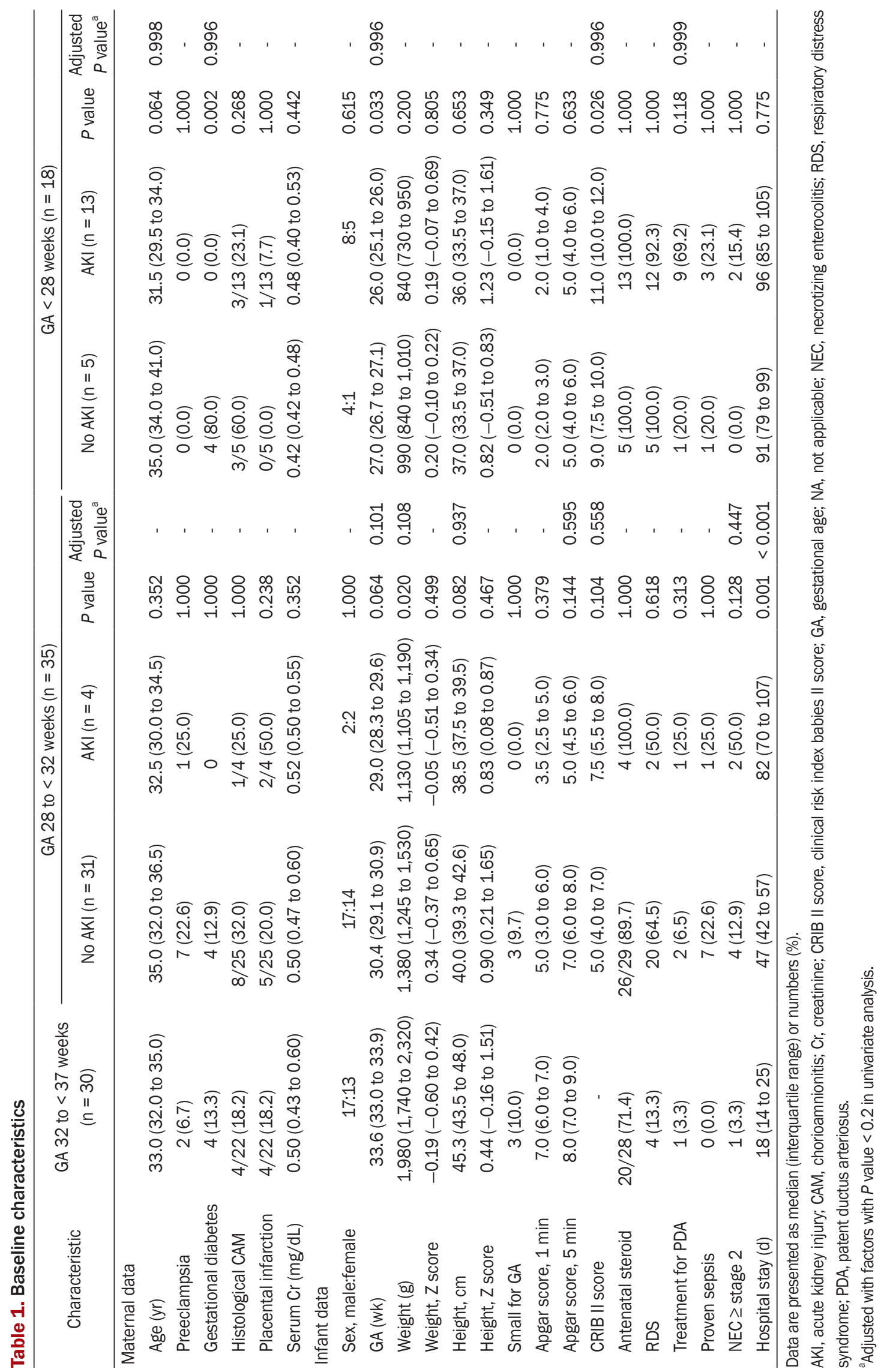


Table 2. Clinical course and kidney outcomes in infants with acute kidney injury

\begin{tabular}{|c|c|c|c|}
\hline & GA 28 to $<32$ weeks $(n=4)$ & $\mathrm{GA}<28$ weeks $(n=13)$ & $P$ value \\
\hline Onset of AKI (d) & $7(5-10)$ & $7(7-9)$ & 0.404 \\
\hline Cause of AKI & & & 0.053 \\
\hline Hemodynamically significant PDA & $1(25.0)$ & $7(53.8)$ & \\
\hline Sepsis & $0(0.0)$ & $2(15.4)$ & \\
\hline Drug-induced & $0(0.0)$ & $3(23.1)$ & \\
\hline NEC & $2(50.0)$ & $0(0.0)$ & \\
\hline Unknown & $1(25.0)^{a}$ & $1(7.7)^{b}$ & \\
\hline AKI stage & & & 0.643 \\
\hline Stage 1 & $3(75.0)$ & $7(53.8)$ & \\
\hline Stage 2 & $1(25.0)$ & $4(30.8)$ & \\
\hline Stage 3 & $0(0.0)$ & $2(15.4)$ & \\
\hline Kidney replacement therapy & $0(0.0)$ & $0(0.0)$ & 1.000 \\
\hline Mortality & $0(0.0)$ & $1(7.7)$ & 1.000 \\
\hline \multicolumn{4}{|l|}{ Renal sequelae at discharge } \\
\hline eGFR, $\mathrm{mL} / \mathrm{min} / 1.73 \mathrm{~m}^{2}$ & $71.6(67.3-107.5)$ & $101.1(57.4-109.0)$ & 0.910 \\
\hline Reduced eGFR & $0(0.0)$ & $0(0.0)$ & 1.000 \\
\hline Proteinuria & $2(50.0)$ & $2(15.4)$ & 0.219 \\
\hline Abnormal findings on USG & $0(0.0)$ & $0(0.0)$ & 1.000 \\
\hline
\end{tabular}

Data are presented as median (interquartile range) or number (\%).

AKI, acute kidney injury; eGFR, estimated glomerular filtration rate; GA, gestational age; NEC, necrotizing enterocolitis; PDA, patent ductus arteriosus; USG, ultrasonography.

${ }^{a}$ This patient had histological chorioamnionitis, and Ureaplasma urealyticum was identified from the first gastric juice, which was regarded as amniotic fluid. The patient also showed a leukemoid reaction for the first 2 weeks of life (white blood cell, 57,000-112,800/ $\mu \mathrm{L}$ ) without any infection focus, which resolved

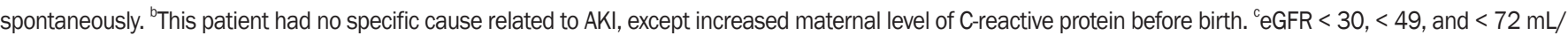
$\mathrm{min} / 1.73 \mathrm{~m}^{2}$ at $<42$ weeks, 42 to $<48$ weeks, and $\geq 48$ weeks of postmenstrual age, respectively.

(no AKI vs. AKI group, median 254 [IQR, 202-275] vs. 473 [309-619] ng/mg at day 3, $P=0.027$; median 278 [IQR, $252-306]$ vs. 384 [343-532] $\mathrm{ng} / \mathrm{mg}$ at day $5, P=0.005$; median 275 [IQR, 121-326] vs. 478 [342-503] ng/mg at day $7, P=0.013$ ). Regarding the timing of AKI (onset at median 7 [IQR, 7-9] days after birth), urine NGAL/Cr was highest before the onset of AKI in all patients except one (the day of highest urine NGAL/Cr - onset day of AKI, median -4 [IQR, -6.5 to -2 ] day). The urine cystatin C/ $\mathrm{Cr}$ levels were significantly higher in the AKI group than in the no AKI group at day 7 (no AKI vs. AKI group, median 1,799 [IQR, $1,090-2,639]$ vs. 5,658 [5,278-15,113] ng/ $\mathrm{mg}, P=0.04)$. Regarding timing with AKI, urine cystatin $\mathrm{C} / \mathrm{Cr}$ was highest at a similar point with the onset of AKI (the day of highest urine cystatin $\mathrm{C} / \mathrm{Cr}$ - onset day of AKI, median 0 [IQR, -3.5 to 1] day). The urine EGF/Cr levels were significantly lower in the AKI group than in the no AKI group at day 5 (no AKI vs. AKI group, median 24.9 [IQR, 23.4-29.6] vs. 16.3 [13.9-22.0] ng/mg, $P=0.019$ ). Regarding the timing of AKI, urine EGF/Cr was lowest at a similar point with the onset of AKI (the day of lowest urine EGF/Cr - onset day of AKI, median 0 [IQR -1.5 to 1.5] day). There were no differences in the levels of other urinary biomarkers between the two groups (Fig. 2).

In infants of GA 28 to $<32$ weeks, serum $\mathrm{Cr}$ levels were not different between the two groups because AKI was defined as decreased urine output $(<0.5 \mathrm{~mL} / \mathrm{kg} / \mathrm{hour}$ for 6 hours) without an increase of serum Cr level in 1 patient and 3 of 4 patients had stage 1 AKI (Table 2, Fig. 3). The urine NGAL/Cr levels were significantly higher in the AKI group than in the no AKI group at day 14 (no AKI vs. AKI group, median 79 [IQR, 37-146] vs. 277 [116437] ng/mg, $P=0.034)$. Regarding timing with AKI, urine NGAL/Cr was highest before the onset of AKI in 2 of 4 patients, but not in the other 2 patients (the day of highest urine NGAL/Cr - onset day of AKI, $-5,-4,3$ and 7 day, respectively). The levels of urine IL-8/Cr were significantly higher in the AKI group than in the no AKI group at days 7 and 10 (no AKI vs. AKI group, median 148 [IQR, $90-339]$ vs. $397[303-5,954] \mathrm{pg} / \mathrm{mg}$ at day $7, P=0.033$; 

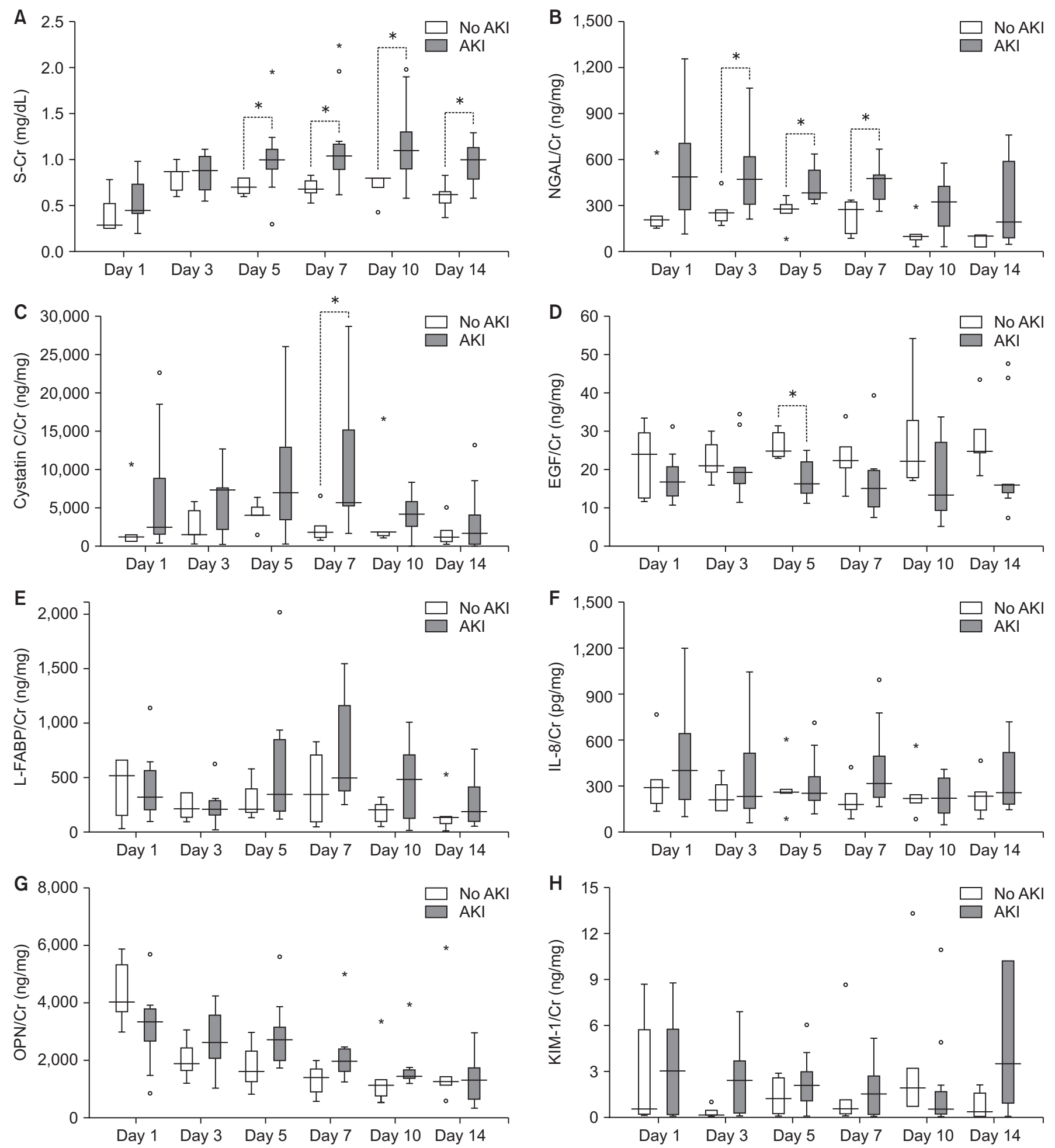

Figure 2. Differences in serum creatinine (S-Cr) and urine biomarkers levels between infants without acute kidney injury (AKI) and with AKI of gestational age < 28 weeks. (A) Change of S-Cr. (B) Change of urine neutrophil-gelatinase-associated lipocalin (NGAL)/Cr ratio. (C) Change of urine cystatin $\mathrm{C} / \mathrm{Cr}$ ratio. (D) Change of urine epidermal growth factor (EGF)/Cr ratio. (E) Change of urine liver fatty acid binding protein (L-FABP)/Cr ratio. (F) Change of urine interleukin-8 (IL-8)/Cr ratio. (G) Change of urine osteopontin (OPN)/Cr ratio. (H) Change of urine kidney injury molecule-1 (KIM-1)/Cr ratio. Values are shown as a box plot with 10, 25, 50, 75 and 90 th percentiles and were analyzed by Mann-Whitney $U$ tests. $* P<0.05$ no AKI group vs. AKI group. 

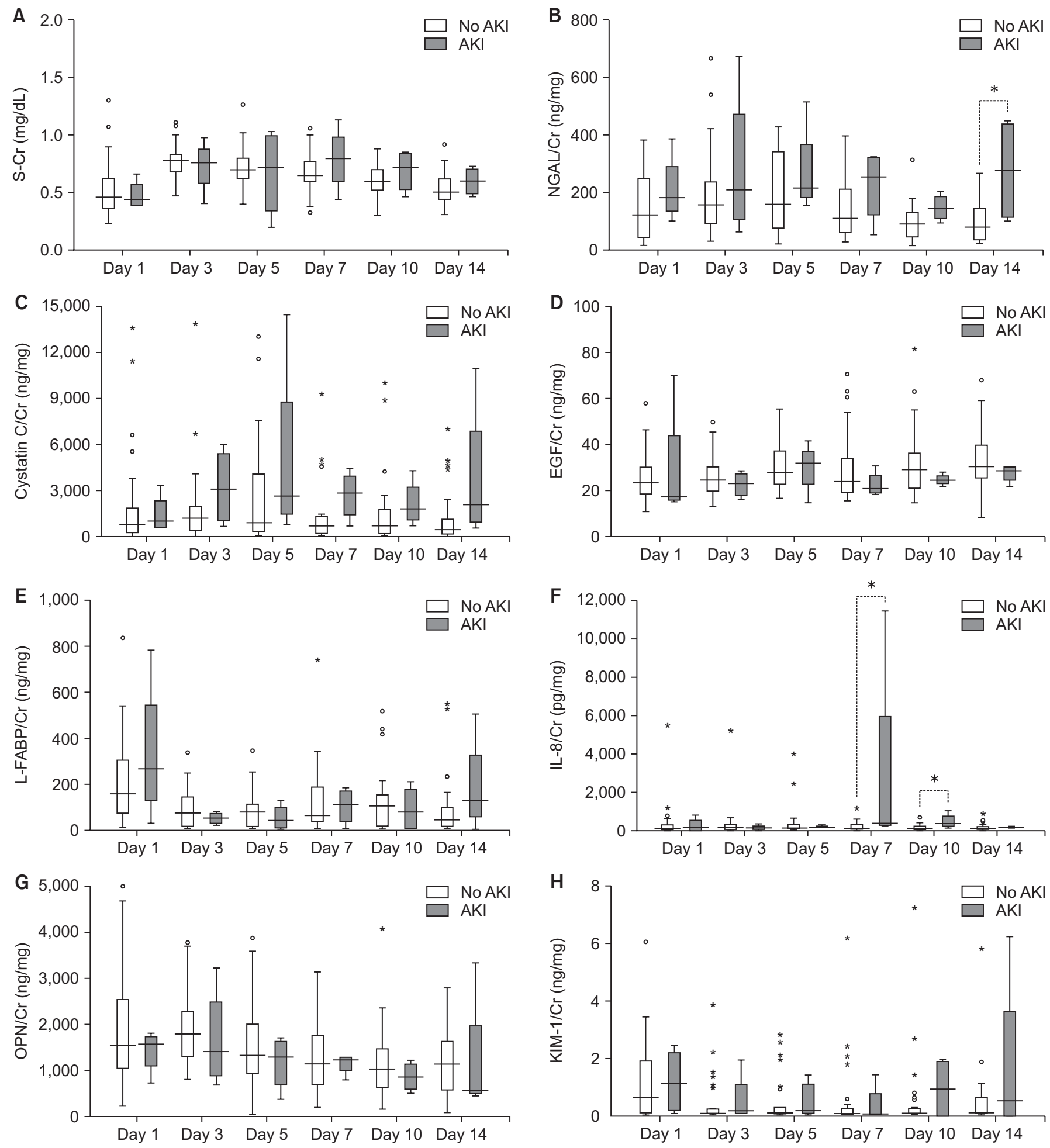

Figure 3. Differences in serum creatinine (S-Cr) and urine biomarker levels between infants without acute kidney injury (AKI) and with AKI of gestational age 28 to $<32$ weeks. (A) Change of S-Cr. (B) Change of urine neutrophil-gelatinase-associated lipocalin (NGAL)/ $\mathrm{Cr}$ ratio. (C) Change of urine cystatin $\mathrm{C} / \mathrm{Cr}$ ratio. (D) Change of urine epidermal growth factor (EGF)/Cr ratio. (E) Change of urine liver fatty acid binding protein (L-FABP)/Cr ratio. (F) Change of urine interleukin-8 (IL-8)/Cr ratio. (G) Change of urine osteopontin (OPN)/Cr ratio. (H) Change of urine kidney injury molecule-1 (KIM-1)/Cr ratio. Values are shown as a box plot with 10, 25, 50, 75 and 90 th percentiles and were analyzed by Mann-Whitney $U$ tests. ${ }^{*} P<0.05$ no AKI group vs. AKI group. 

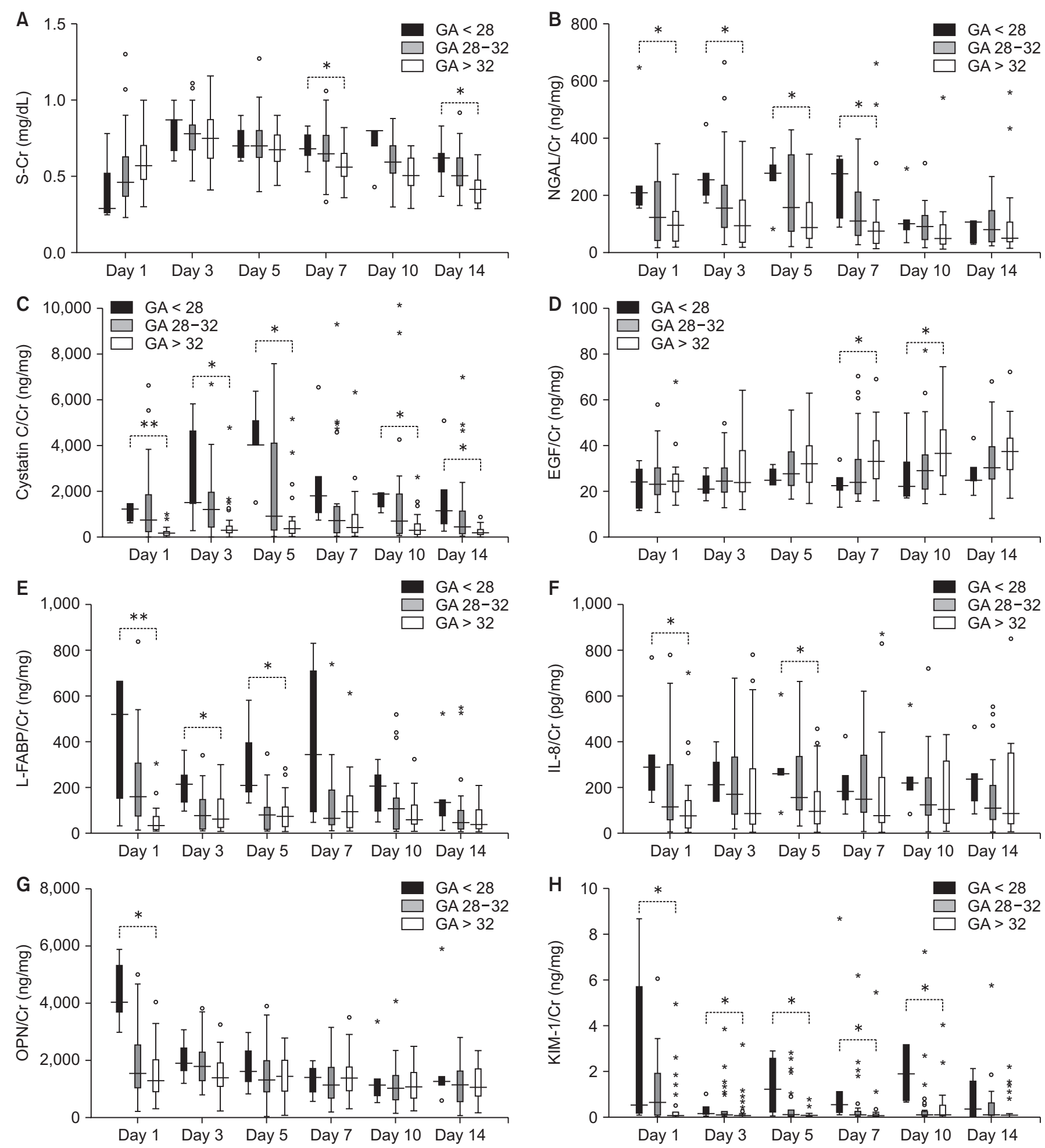

Figure 4. Differences in serum creatinine (S-Cr) and urine biomarkers levels according to gestational age (GA) in infants without acute kidney injury. (A) Change of S-Cr. (B) Change of urine neutrophil-gelatinase-associated lipocalin (NGAL)/Cr ratio. (C) Change of urine cystatin $\mathrm{C} / \mathrm{Cr}$ ratio. (D) Change of urine epidermal growth factor (EGF)/Cr ratio. (E) Change of urine liver fatty acid binding protein (L-FABP)/Cr ratio. (F) Change of urine interleukin-8 (IL-8)/Cr ratio. (G) Change of urine osteopontin (OPN)/Cr ratio. (H) Change of urine kidney injury molecule-1 (KIM-1)/Cr ratio. Values are shown in a box plot with 10, 25, 50, 75 and 90th percentiles and were analyzed by the Kruskal-Wallis test. $* P<0.05, * * P<0.001$ between groups. 
median 124 [IQR, 78-240] vs. 378 [241-745] pg/mg at day $10, P=0.023$, respectively). Regarding the timing with AKI, urine IL-8/Cr was highest before or at the onset of AKI in all patients (the day of highest urine IL-8/Cr - onset day of AKI, $-6,-3,0$ and 0 day, respectively). There were no differences in the levels of other urine biomarkers between the two groups.

Among infants without AKI, urine biomarker levels were different according to GA groups (Fig. 4). Consistent with previous reports [14-16], urinary levels of NGAL/Cr, cystatin $\mathrm{C} / \mathrm{Cr}$, L-FABP/Cr and $\mathrm{KIM}-1 / \mathrm{Cr}$ were inversely correlated with GA, especially during the first week of life. In contrast, urinary EGF/Cr levels increased significantly with GA at postnatal days 7 and 10 in this study. In addition, the urinary levels of NGAL, cystatin $\mathrm{C}$ and LFABP decreased after 1 week of life compared to the first postnatal week. Urinary OPN/Cr levels were not affected by GA or the day after birth.

\section{Discussion}

In this prospective cohort study, we found that urinary biomarkers associated with the development of AKI differed according to the GA. While urinary NGAL levels were significantly higher prior to the onset of AKI among infants of GA $<28$ weeks, they did not differ prior to AKI in infants of GA 28 to $<32$ weeks. Similarly, while urinary cystatin C and EGF levels were significantly different at the onset of AKI between groups among infants of GA < 28 weeks, they did not differ between groups in infants of GA 28 to $<32$ weeks. The urinary IL-8 levels were significantly higher in the AKI group before or at the onset of AKI only in infants of GA of 28 to $<32$ weeks.

Some studies have shown urinary and serum NGAL levels can be useful as predictive markers of AKI in premature infants [17-19]. On the other hand, one pilot study performed among premature infants with a mean GA of 29.6 weeks reported that urine NGAL levels were associated with AKI but failed to predict AKI [20]. According to a recently published case-control study based on a prospective cohort of premature infants (mean GA 25.2 weeks), urinary NGAL and EGF levels predict AKI development prior to changes in serum $\mathrm{Cr}$ [19]. The differences among results of previous studies may be due to differences in the GA of the infants included in these studies. Our study also showed that urinary NGAL levels were meaningful as prediction markers in infants born before GA 28 weeks but not in infants of GA 28 to $<32$ weeks. In infants of GA $<28$ weeks, serum $\mathrm{Cr}$ differed between the AKI group and the no AKI group from the fifth day after birth, but NGAL/Cr begins to differ from the third day after birth (Fig. 1). Therefore, urinary NGAL/Cr could be an early biomarker to diagnose AKI in extremely premature infants.

AKI is a very complex, heterogeneous disorder that occurs with varying degrees of severity in different clinical situations. Our results support the hypothesis that the pathophysiology of AKI in premature infants depends on immaturity and the urinary biomarkers examined and according to GA. Extremely preterm infants born before GA 28 weeks have few nephrons or very immature nephrons [21]. Their postnatal kidney maturation can be accelerated or arrested by various factors in the extrauterine environment $[5,22]$. In extremely premature infants, the most important mechanism of AKI would be related to the disruption or arrest of normal maturation of the kidneys. The significantly lower levels of urinary EGF in infants with AKI could be interpreted in a similar manner. One longitudinal investigation of urine biomarkers in preterm infants demonstrated that urine EGF levels increased as kidney maturation progressed with advancing gestation [23]. EGF is a growth factor that stimulates cell growth, proliferation, and differentiation by binding to its receptor. Experimental studies showed that the activation of EGF receptor located on proximal tubules is essential for kidney development and recovery from AKI $[23,24]$.

Interestingly, the levels of urinary IL-8 were associated with AKI in infants of GA 28 to $<32$ weeks. IL-8 is a powerful chemokine involved in tissue neutrophil recruitment. The high levels of IL-8 in AKI group reflect increased inflammation related to the development of AKI in this group [25-27]. Systemic inflammation frequently occurred in premature infants, including bacterial sepsis and NEC. Although the incidence of sepsis was not different between groups, $50 \%$ of infants with AKI had stage 2 or higher NEC (vs. $12.9 \%$ in the no AKI group) among infants of GA of 28 to $<32$ weeks (Table 2). One patient classified as having an unknown cause of AKI had histological chorioamnionitis, and Ureaplasma urealyticum was identified in the first gastric juice, which was regarded to have originated as amniotic fluid. This patient also showed a leukemoid reaction for the first 2 weeks of life 
(white blood cell, 57,000-112,800/ $\mu \mathrm{L}$ ) without any infection focus, which resolved spontaneously. In an animal model of AKI, inflammatory cytokines including keratinocyte-derived chemokine (KC, the murine analogue of IL-8) increased early in the process of AKI [28]. In addition, KC/IL-8 receptor knockout mice were protected against colitis-induced AKI and inflammation [27]. According to these findings, inflammation might be a major cause of AKI in premature infants born after 28 weeks of gestation.

Askenazi et al [15] reported that the urine levels of NGAL, KIM-1, OPN, and cystatin C decreased with increasing GA and suggested that those findings should be considered when analyzing biomarkers in newborns with AKI. Our results support this hypothesis. In this study, urinary levels of NGAL, KIM-1, cystatin C, L-FABP, and IL-8 were inversely associated with GA in infants without AKI (Fig. 4). In contrast, urinary EGF/Cr levels increased significantly with GA after 1 week of birth. Because nephrogenesis is not complete but rather is ongoing, urinary biomarkers should be evaluated and used after stratification for gestation and postnatal day in preterm infants.

Several factors need to be considered as limitations in this study. To define AKI, we defined the lowest serum Cr level after the 2nd day of life as the baseline value because neonatal serum $\mathrm{Cr}$ on the first day of life reflects maternal levels, and diuresis usually occurs within 2 to 3 days after birth. Indeed, initial neonatal serum $\mathrm{Cr} \mathrm{lev-}$ els were significantly correlated with maternal serum $\mathrm{Cr}$ levels in our sample (Pearson's correlation $r=0.556, P<$ 0.001 ). However, serum $\mathrm{Cr}$ after the 2nd day of life may also be affected by several co-morbidities in premature infants that impact their hemodynamic status, such as PDA, intraventricular hemorrhage, and ventilator support. Second, as the stratification for GA and lack of AKI was determined in infants of GA > 32 weeks, the number of infants actually included in the analysis was reduced from the original 83 infants to 53 . Because premature infants born after GA 32 weeks are at low risk for AKI, future studies of urinary biomarkers for AKI should focus on very premature infants born before GA 32 weeks. Third, the number of AKI patients in each GA group was small. A larger prospective study could provide more meaningful results about urine biomarkers for AKI in premature infants.
In conclusion, several urine biomarkers were significantly different between infants with and without AKI and, and some of the biomarkers had changed before the onset of AKI in premature infants. Which urine biomarkers are meaningful may differ according to the causative factors of AKI and GA. Urine biomarkers could be useful for predicting the development of AKI when interpreted appropriately according to the clinical situation.

\section{Conflicts of interest}

All authors have no conflicts of interest to declare.

\section{Funding}

This research was supported by the Hallym University Research Fund in 2016 (HURF-2016-11) and an Inha University Research Grant (INHA-53356).

\section{Acknowledgments}

We thank all of the NICU residents and nurses who participated in collecting samples.

\section{Authors' contributions}

Yo Han Ahn, Juyoung Lee, and Tae-Jung Sung conceived and designed the study. Yo Han Ahn, Juyoung Lee, and Jiyoung Chun participated in data collection. Yo Han Ahn, Juyoung Lee, Jiyoung Chun, Yong Hoon Jun, and TaeJung Sung performed the study. Yo Han Ahn and Juyoung Lee performed statistical analysis and interpretation of data. Yo Han Ahn, Juyoung Lee, and Tae-Jung Sung wrote the manuscript. Tae-Jung Sung and Yong Hoon Jun revised the manuscript. All authors read and approved the final manuscript.

\section{References}

[1] Stojanović V, Barišić N, Milanović B, Doronjski A. Acute kidney injury in preterm infants admitted to a neonatal intensive care unit. Pediatr Nephrol 2014;29:2213-2220.

[2] Koralkar R, Ambalavanan N, Levitan EB, McGwin G, Goldstein S, Askenazi D. Acute kidney injury reduces survival in very low birth weight infants. Pediatr Res 2011;69:354-358.

[3] Carmody JB, Swanson JR, Rhone ET, Charlton JR. Recogni- 
tion and reporting of AKI in very low birth weight infants. Clin J Am Soc Nephrol 2014;9:2036-2043.

[4] Viswanathan S, Manyam B, Azhibekov T, Mhanna MJ. Risk factors associated with acute kidney injury in extremely low birth weight (ELBW) infants. Pediatr Nephrol 2012;27:303311.

[5] Sutherland MR, Gubhaju L, Moore L, et al. Accelerated maturation and abnormal morphology in the preterm neonatal kidney. J Am Soc Nephrol 2011;22:1365-1374.

[6] Selewski DT, Charlton JR, Jetton JG, et al. Neonatal acute kidney injury. Pediatrics 2015;136:e463-e473.

[7] Carmody JB, Charlton JR. Short-term gestation, long-term risk: prematurity and chronic kidney disease. Pediatrics 2013;131:1168-1179.

[8] White SL, Perkovic V, Cass A, et al. Is low birth weight an antecedent of CKD in later life? A systematic review of observational studies. Am J Kidney Dis 2009;54:248-261.

[9] Bertram JF, Douglas-Denton RN, Diouf B, Hughson MD, Hoy WE. Human nephron number: implications for health and disease. Pediatr Nephrol 2011;26:1529-1533.

[10] Luyckx VA, Bertram JF, Brenner BM, et al. Effect of fetal and child health on kidney development and long-term risk of hypertension and kidney disease. Lancet 2013;382:273-283.

[11] Abitbol CL, DeFreitas MJ, Strauss J. Assessment of kidney function in preterm infants: lifelong implications. Pediatr Nephrol 2016;31:2213-2222.

[12] Parry G, Tucker J, Tarnow-Mordi W; UK Neonatal Staffing Study Collaborative Group. CRIB II: an update of the clinical risk index for babies score. Lancet 2003;361:1789-1791.

[13] Kang HG, Choi HJ, Han KH, et al. KNOW-Ped CKD (KoreaN cohort study for outcomes in patients with pediatric CKD): design and methods. BMC Nephrol 2016;17:35.

[14] Gubhaju L, Sutherland MR, Horne RS, et al. Assessment of renal functional maturation and injury in preterm neonates during the first month of life. Am J Physiol Renal Physiol 2014;307:F149-F158.

[15] Askenazi DJ, Koralkar R, Levitan EB, et al. Baseline values of candidate urine acute kidney injury biomarkers vary by gestational age in premature infants. Pediatr Res 2011;70: 302-306.

[16] Elmas AT, Tabel Y, Ipek S. Determination of reference values for urinary neutrophil gelatinase-associated lipocalin in premature infants. J Matern Fetal Neonatal Med 2014; 27:187-191.

[17] Tabel Y, Elmas A, Ipek S, Karadag A, Elmas O, Ozyalin F. Urinary neutrophil gelatinase-associated lipocalin as an early biomarker for prediction of acute kidney injury in preterm infants. Am J Perinatol 2014;31:167-174.

[18] Pejović B, Erić-Marinković J, Pejović M, Kotur-Stevuljević J, Peco-Antić A. Detection of acute kidney injury in premature asphyxiated neonates by serum neutrophil gelatinaseassociated lipocalin (sNGAL)--sensitivity and specificity of a potential new biomarker. Biochem Med (Zagreb) 2015; 25:450-459.

[19] Hanna M, Brophy PD, Giannone PJ, Joshi MS, Bauer JA, RamachandraRao S. Early urinary biomarkers of acute kidney injury in preterm infants. Pediatr Res 2016;80:218-223.

[20] Sarafidis K, Tsepkentzi E, Diamanti E, et al. Urine neutrophil gelatinase-associated lipocalin to predict acute kidney injury in preterm neonates. A pilot study. Pediatr Nephrol 2014;29:305-310.

[21] Hinchliffe SA, Sargent PH, Howard CV, Chan YF, van Velzen D. Human intrauterine renal growth expressed in absolute number of glomeruli assessed by the disector method and Cavalieri principle. Lab Invest 1991;64:777-784.

[22] Rodríguez MM, Gómez AH, Abitbol CL, Chandar JJ, Duara S, Zilleruelo GE. Histomorphometric analysis of postnatal glomerulogenesis in extremely preterm infants. Pediatr Dev Pathol 2004;7:17-25.

[23] Wang Z, Chen JK, Wang SW, Moeckel G, Harris RC. Importance of functional EGF receptors in recovery from acute nephrotoxic injury. J Am Soc Nephrol 2003;14:3147-3154.

[24] Chen J, Chen JK, Harris RC. Deletion of the epidermal growth factor receptor in renal proximal tubule epithelial cells delays recovery from acute kidney injury. Version 2. Kidney Int 2012;82:45-52.

[25] Liangos O, Kolyada A, Tighiouart H, Perianayagam MC, Wald R, Jaber BL. Interleukin-8 and acute kidney injury following cardiopulmonary bypass: a prospective cohort study. Nephron Clin Pract 2009;113:c148-c154.

[26] Kwon O, Molitoris BA, Pescovitz M, Kelly KJ. Urinary actin, interleukin-6, and interleukin-8 may predict sustained ARF after ischemic injury in renal allografts. Am J Kidney Dis 2003;41:1074-1087.

[27] Ranganathan P, Jayakumar C, Manicassamy S, Ramesh G. CXCR2 knockout mice are protected against DSS-colitis-induced acute kidney injury and inflammation. Am J Physiol Renal Physiol 2013;305:F1422-F1427.

[28] Hoke TS, Douglas IS, Klein CL, et al. Acute renal failure after bilateral nephrectomy is associated with cytokinemediated pulmonary injury. J Am Soc Nephrol 2007;18:155164. 